\title{
Topological Dirac nodal loops in nonsymmorphic hydrogenated monolayer boron
}

\author{
N. T. Cuong $\odot,{ }^{1}$ I. Tateishi $\odot,{ }^{2,}{ }^{*}$ M. Cameau $\odot,{ }^{3}$ M. Niibe $\odot,{ }^{4,5}$ N. Umezawa $\odot,{ }^{6}$ B. Slater, ${ }^{7}$ K. Yubuta $\odot,{ }^{8}$ \\ T. Kondo $\odot,{ }^{1,9}$ M. Ogata, ${ }^{2}$ S. Okada, ${ }^{1}$ and I. Matsuda $\odot^{5, \dagger}$ \\ ${ }^{1}$ Faculty of Pure and Applied Sciences, University of Tsukuba, Tsukuba, 305-8571, Japan \\ ${ }^{2}$ Department of Physics, School of Science, University of Tokyo, Hongo, Tokyo 113-0033, Japan \\ ${ }^{3}$ Institut des Nanosciences de Paris, Sorbonne Universite, Paris, 75005, France \\ ${ }^{4}$ Laboratory of Advanced Science and Technology for Industry (LASTI), University of Hyogo, Hyogo 678-1205, Japan \\ ${ }^{5}$ Institute for Solid State Physics, The University of Tokyo, Kashiwa, Chiba 277-8581, Japan \\ ${ }^{6}$ Semiconductor R\&D Center, Samsung Electronics, 1, Samsungjeonja-ro, Hwaseong-si, Gyeonggi-do 18448, Korea \\ ${ }^{7}$ Department of Chemistry, University College London, 20 Gordon Street, London WC1H OAJ, United Kingdom \\ ${ }^{8}$ Institute for Materials Research, Tohoku University, Sendai 980-8577, Japan \\ ${ }^{9}$ Materials Research Center for Element Strategy, Tokyo Institute of Technology, Yokohama 226-8503, Japan
}

(Received 30 June 2019; revised manuscript received 26 March 2020; accepted 27 March 2020; published 7 May 2020)

\begin{abstract}
The existence of a topological Dirac nodal loop is predicted for hydrogenated monolayer boron sheets with a nonsymmorphic symmetry. The three-center two-electron bonds in boron compounds restrict the electronic system to be insulating or semimetallic with a Dirac nodal loop. Two types of electronic structures are distinguished by the $\mathbb{Z}_{2}$ topological index and confirmed by first-principles total-energy calculations. The topological taxonomy, developed in this research, can be applied to other two-dimensional materials and to seek novel Dirac fermions.
\end{abstract}

DOI: 10.1103/PhysRevB.101.195412

\section{INTRODUCTION}

Two-dimensional (2D) materials containing Dirac fermions have recently attracted intensive research attention for pursuing fundamental science and developing quantum devices [1-3]. Research on free-standing graphene, an archetype, has revealed the basic physics of Dirac fermions and revolutionized our nanotechnology which motivates further work to synthesize new 2D materials with potential novel electronic structure. Massive and massless Dirac fermions have been reported in the form of Dirac bands in atomic layers of Xenes (i.e., silicene [4-7], germanene [8,9], and borophene [10-12]). When the valence and conduction bands in Dirac fermion materials touch at extended and closed rings, novel Dirac nodal loops (DNLs) quantum states emerge and could exhibit intriguing physical properties, such as ultrahigh carrier mobility or unique spin transport properties [13,14]. Therefore 2D materials with DNL fermions are now the focus of this field to realize a new and exciting platform for exploring the exotic properties of Dirac fermions and designing of novel quantum electronic devices at the nanoscale.

Recently, DNL fermions have been experimentally observed in several bulk materials, such as $\mathrm{PtSn}_{4}$ [15], $\mathrm{PbTaSe}_{2}$ [16], ZrSiS [17], and InBi [18]. However, experimental observation of DNL fermions in 2D materials is still lacking, excepting recent works on monolayer $\mathrm{Cu}_{2} \mathrm{Si}$ [19] or

\footnotetext{
*i.tateishi@hosi.phys.s.u-tokyo.ac.jp

†imatsuda@issp.u-tokyo.ac.jp
}

CuSe [20] on the $\mathrm{Cu}(111)$ substrate. Therefore 2D materials with DNL fermions are now the focus of this field [21,22]. On the other hand, those monatomic layers have Dirac fermions but are mostly restricted to prepare on a solid substrate. Moreover, free-standing 2D materials are much more convenient in experiments and applications. The search for new and feasible synthetic free-standing 2D materials with DNL fermions have attracted broad interests in condensed matter physics.

In this paper, we predict the existence of DNLs in freestanding layers of hydrogenated monolayer boron sheets (borophane or HB) based on the topological band theory and first-principles calculations based on density functional theory. The DNLs are topologically protected by the local chemical bonding and nonsymmorphic symmetry. The threecenter two-electron bonds in the HB sheets restrict the electronic system to be semimetallic with a nodal loop at the Fermi level or insulating. Two types of electronic structures are distinguished by a $\mathbb{Z}_{2}$ topological index. Moreover, the results are confirmed by band calculation using the density functional theory. The present topological classification can also be applied to the other atomic sheets to identify novel Dirac fermions.

\section{BOROPHANE}

\section{A. (5-7)- $\alpha$-borophane}

Figure 1(a) shows a structure model of a monatomic boron network [(5-7)- $\alpha$-borophene] that is composed of 5membered and 7-membered rings of boron atoms. This (5-7)borophene layer, labeled $\alpha$, is found in crystals of metal boride, such as $\mathrm{TmAlB}_{4}$ [23]. The structure belongs to a 

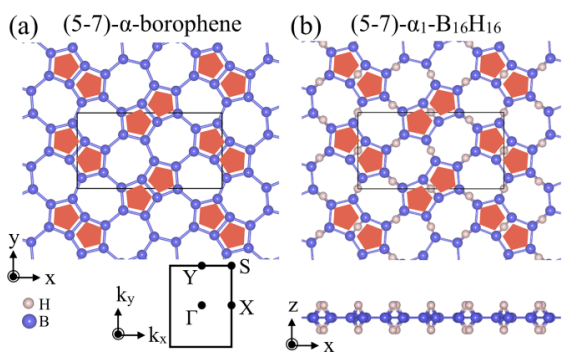

(c) (5-7)- $\alpha_{2}-\mathrm{B}_{16} \mathrm{H}_{16}$



FIG. 1. (a) Atomic structure of (5-7)- $\alpha$-borophene monolayer, and two-dimensional Brillouin zone with symbols at the symmetry points. Top and side views of atomic structures of the HB layer (borophane) of (b) (5-7)- $\alpha_{1}-\mathrm{B}_{16} \mathrm{H}_{16}$ monolayer and (c) (5-7)- $\alpha_{2}-\mathrm{B}_{16} \mathrm{H}_{16}$ monolayer. Boron and hydrogen atoms are shown as blue and white balls, respectively, while the unit cell is shown by the solid line. Locations of the 5-membered rings are indicated with red pentagons. Bond drawings are defined by the interatomic distance.

nonsymmorphic space group, Pbam (the layer group No. 44 or the 3D space group No. 55). The two-dimensional Brillouin zone is illustrated in Fig. 1(a) with symbols at the highsymmetry points. More detailed properties of this space group are provided in the coming section.

Hydrogenation of the $\alpha$-borophene layer results in the formation of (5-7)- $\alpha_{1}-\mathrm{B}_{16} \mathrm{H}_{16}$ and (5-7)- $\alpha_{2}-\mathrm{B}_{16} \mathrm{H}_{16}$ borophane (HB) sheets, in which the B-H-B bonds are the three-center two-electron bonds [24-29], as realized in the diborane $\left(\mathrm{B}_{2} \mathrm{H}_{6}\right)$ molecule. The layer structure is an assembly of the building blocks of $\mathrm{B}_{2} \mathrm{H}_{2}$ that have even number of electrons, $2 n\left(N_{\mathrm{B}}+N_{\mathrm{H}}\right)=12 n$. Here, $N_{\mathrm{B}}$ and $N_{\mathrm{H}}$ are numbers of valence electrons of a boron atom $\left(N_{\mathrm{B}}=5\right)$ and a hydrogen atom $\left(N_{\mathrm{H}}=1\right)$, respectively, while $n$ is a natural number and it represents a number of the $\mathrm{B}_{2} \mathrm{H}_{2}$ blocks. The $\mathrm{B}-\mathrm{H}-\mathrm{B}$ bonding motifs are established at both sides $(+z,-z)$ of the boron layer. Since the 2D crystal structure keeps the mirror-plane reflection symmetry, the (5-7)- $\alpha$-borophane layers conserve the symmetries of (5-7)- $\alpha$-borophene [the layer group No. $44]$, when the bonding configurations are arranged as the $\alpha_{1}$ model [Fig. 1(b)] or the $\alpha_{2}$ model [Fig. 1(c)]. Due to alternative locations of $\mathrm{B}-\mathrm{B}$ bonds, the bonding configurations are inverted between the $\alpha_{1}$ and $\alpha_{2}$ models. It should be noted that the nonsymmorphic symmetry itself also restricts the number of atoms in a unit cell to be even.

\section{B. (5-6-7)- $\gamma$-borophane}

There are other HB sheets that keep the symmetry of the layer group No. 44. As shown in Fig. 2(a), the (5-6-7)borophene layer, named $\gamma$, is composed of 5-membered, 6membered, and 7-membered rings of boron atoms that is found in crystals of metal boride, such as $\mathrm{Tm}_{2} \mathrm{AlB}_{6}$ [23]. This boron structure also belongs to the layer group No. 44. In the (5-6-7)- $\gamma$-borophane, hydrogenations take the four bonding configurations: $\gamma_{1^{-}}, \gamma_{2^{-}}, \gamma_{3^{-}}$, and $\gamma_{4}-\mathrm{B}_{24} \mathrm{H}_{24}$ (see all atomic geometries in Appendix F). Two of them, the $\gamma_{1}$ and $\gamma_{2}$ configurations keep the layer group No. 44, as shown in Figs. 2(b) and 2(c), while the others belong to the different symmetry, demonstrating the symmetry restriction by the heteroatom adsorption [30,31]. (a) (5-6-7)- $\gamma$-borophene

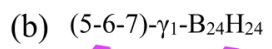

(c) $(5-6-7)-\gamma_{2}-\mathrm{B}_{24} \mathrm{H}_{24}$
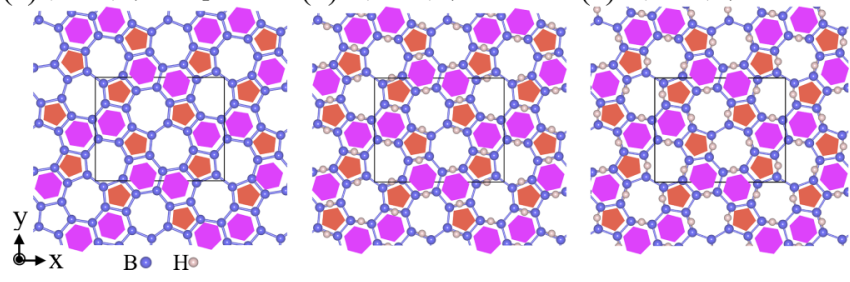

FIG. 2. (a) Atomic structure of (5-6-7)- $\gamma$-borophene, (b) the HB layer (borophane) of (5-6-7)- $\gamma_{1}-\mathrm{B}_{24} \mathrm{H}_{24}$, and (c) the HB layer (borophane) of (5-6-7)- $\gamma_{2}-\mathrm{B}_{24} \mathrm{H}_{24}$. Locations of the 5-membered and 6-membered rings are indicated with red pentagons and purple hexagons, respectively.

\section{THEORY}

\section{A. Nonsymmorphic symmetry and $\mathbb{Z}_{2}$ index}

Nonsymmorphic symmetries generally give nontrivial band structures on the boundary of the Brillouin zone. Here we focus on space group Pbam (the 3D space group No. 55 or the layer group No. 44) and discuss its properties of band dispersion by using the representation theory [32].

First of all, we note the relation between the $3 \mathrm{D}$ space group and the layer group. The 3D space group consists of 3D translation and $3 \mathrm{D}$ point group operation and they are used to describe the symmetries of 3D crystals. For 2D materials, the wave functions of electrons have a 3D structure. Therefore the $2 \mathrm{D}$ space group, which consists of $2 \mathrm{D}$ translation and $2 \mathrm{D}$ point group operation, is not enough to describe 2D materials. The layer group, which consists of $2 \mathrm{D}$ translation and $3 \mathrm{D}$ point group operation, should be used. A layer group is equivalent to a 3D space group with an axis of infinite length. For example, the layer group No. 44 (Pbam) is equivalent to the space group No. 55 with the infinitely long $c$ axis.

\section{General properties of the layer group No. 44 (Pbam)}

The elements of the layer group No. 44 (Pbam) are written as

$$
\begin{aligned}
& \left\{\{E \mid 000\},\left\{C_{2 z} \mid 000\right\},\left\{C_{2 x} \mid \frac{1}{2} \frac{1}{2} 0\right\},\left\{C_{2 y} \mid \frac{1}{2} \frac{1}{2} 0\right\},\right. \\
& \left.\{I \mid 000\},\left\{\sigma_{z} \mid 000\right\},\left\{\sigma_{x} \mid \frac{1}{2} \frac{1}{2} 0\right\},\left\{\sigma_{y} \mid \frac{1}{2} \frac{1}{2} 0\right\}\right\} \otimes \boldsymbol{T},
\end{aligned}
$$

where $\boldsymbol{T}$ is basic translations

$$
\boldsymbol{T}=\left\{t_{1} \boldsymbol{a}_{1}+t_{2} \boldsymbol{a}_{2} \mid t_{1}, t_{2} \in \mathbb{Z}\right\}
$$

and the element $\left\{C_{2 x} \mid \frac{1}{2} \frac{1}{2} 0\right\}$ acts as

$$
\left\{C_{2 x} \mid \frac{1}{2} \frac{1}{2} 0\right\} \boldsymbol{r}=C_{2 x} \boldsymbol{r}+\frac{1}{2} \boldsymbol{a}_{1}+\frac{1}{2} \boldsymbol{a}_{2}
$$

(see Fig. 3). Here, $\boldsymbol{a}_{1}$ and $\boldsymbol{a}_{2}$ are the basic translation vectors. $E, C_{2 z}, I$, and $\sigma_{z}$ are identical operator, twofold rotation around $z$ axis, inversion, and mirror operation for the $x-y$ plane, respectively.

The Brillouin zone of the layer group No. 44 is depicted in Fig. 4. The character tables of the single-valued irreducible representations of the little group of each $k$ point are shown in Tables I- IV. In the $\Gamma$ point, all irreducible representations are one-dimensional representations and there is no symmetry protected degeneracy. Meanwhile, in the $X$ and $Y$ points, there are only two-dimensional representations and in the $C, D$, 
(a)

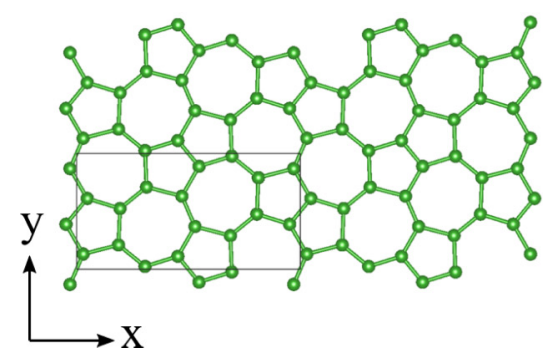

(b)

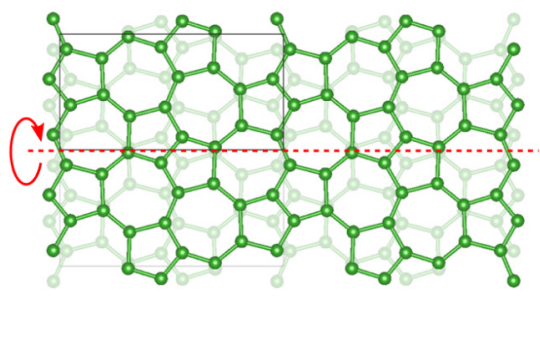

(c)

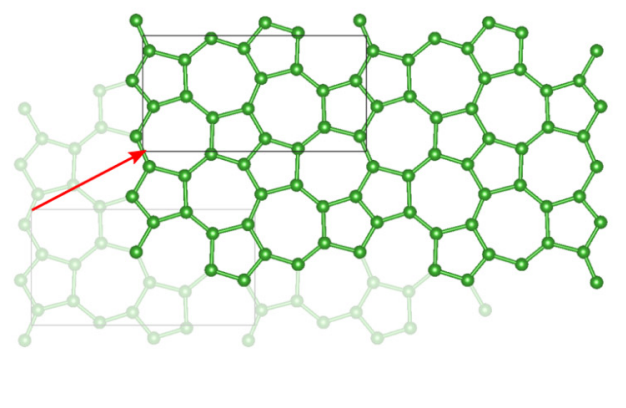

FIG. 3. (a) $\alpha$-borophene as an example of the layer group No. 44. (b) (5-7)- $\alpha$-borophene before (translucent) and after (opaque) $C_{2 x}$ operation. (c) $\alpha$-borophene translated by $\frac{1}{2} \boldsymbol{a}_{1}+\frac{1}{2} \boldsymbol{a}_{2}$ after (b). We can see that now the new configuration (opaque) is equivalent to the original one in (a) (shown as the translucent one).

and $S$ points effectively two-dimensional representations are allowed under the time-reversal symmetry. This fact means that there are only doubly degenerate bands on the zone boundary. Let us focus on the character ( $\simeq$ eigenvalue) of $\left\{\sigma_{z} \mid 000\right\}$. In the $X$ and $Y$ points, the character of $\left\{\sigma_{z} \mid 000\right\}$ is 2 or -2 . It means that the doubly degenerate pair is a pair of two bands with $+1\left\{\sigma_{z} \mid 000\right\}$ eigenvalues or a pair of two bands with $-1\left\{\sigma_{z} \mid 000\right\}$ eigenvalues (a schematic picture is shown in Fig. 5).

The layer group No. $63(P 4 / m b m, 3 \mathrm{D}$ space group No. 127) has the same property, only doubly degenerate bands with the same $\left\{\sigma_{z} \mid 000\right\}$ eigenvalues can exist on the zone boundary. For example, the Shastry-Sutherland lattice [33] has the symmetry of the layer group No. 63. We checked all 80 layer groups and it is found that only the layer group Nos. 44 and 63 have this property.

\section{Application for the $\mathrm{HB}$ sheets}

(5-7)- $\alpha_{1}-\mathrm{B}_{16} \mathrm{H}_{16}$, (5-7)- $\alpha_{2}-\mathrm{B}_{16} \mathrm{H}_{16}$, (5-6-7)- $\gamma_{1}-\mathrm{B}_{24} \mathrm{H}_{24}$, and (5-6-7) $-\gamma_{2}-\mathrm{B}_{24} \mathrm{H}_{24}$ have the symmetry of the layer group No. 44. The filling factor of a HB sheet is $4 n^{\prime}$, as determined by symmetry (see Appendix A). Now we turn our attentions to the band dispersion of $\mathrm{HB}$ sheets. On the zone boundary, there are only doubly degenerate bands. Remembering that we



FIG. 4. Brillouin zone of the layer group No. 44. neglected the spin degree of freedom, the doubly degenerate pair contains four electrons. When the system is not metallic, these facts require the doubly degenerate pair on the zone boundary to be completely occupied or completely unoccupied and a half occupied pair is prohibited. Because each degenerate pair is a pair with the same $\left\{\sigma_{z} \mid 000\right\}$ eigenvalue, the number of occupied bands with $+1(-1)$ eigenvalue is always an even number on the zone boundary.

For the $\Gamma$ point, the number of occupied bands should be the same as that on zone boundary in a nonmetallic system, otherwise, there must be a band crossing the Fermi level and it makes the system metallic. Contrary to the zone boundary, on the $\Gamma$ point, there is no symmetry protected degeneracy and the number of the occupied bands with $+1(-1)$ eigenvalues is not necessarily an even number. When the number of the occupied bands with $+1(-1)$ eigenvalues is an odd number, we can predict a nontrivial band structure in the system. All $\boldsymbol{k}$ points in the 2D Brillouin zone is the $\left\{\sigma_{z} \mid 000\right\}$ invariant point (plane) and thus the $\left\{\sigma_{z} \mid 000\right\}$ eigenvalue is well-defined there. A continuous band must have the same eigenvalue in all $\boldsymbol{k}$ points. If the number of bands with +1 eigenvalues is an odd number on the $\Gamma$ point, there must be a band inversion between the $\Gamma$ point and the zone boundary because that on the zone boundary is known to be an even number. At the same time, the band inversion should exchange bands with +1 eigenvalue and with -1 eigenvalue. A hybridization between bands with different symmetry is generally prohibited and the band inversion will result in gapless band crossing. Now the band crossing is protected by $\left\{\sigma_{z} \mid 000\right\}$ symmetry and thus the same statement is true for all directions from the $\Gamma$ point. Therefore the band crossing is a Dirac nodal loop.

As a consequence, whether the number of bands with +1 $(-1)$ eigenvalues is an even or an odd number on the $\Gamma$ point is an indicator of the existence of a Dirac nodal loop. Then the formula

$$
\mathbb{Z}_{2}=\prod_{\text {occupied }} \Gamma_{i}\left(\sigma_{z}\right)=\left\{\begin{array}{cc}
1 & (\text { even }: 0,2,4, \ldots) \\
-1 & (\text { odd }: 1,3,5, \ldots)
\end{array}\right.
$$

can distinguish them. Here $\sigma_{z}$ is the shorthand of $\left\{\sigma_{z} \mid 000\right\}$ and $\Gamma_{i}\left(\sigma_{z}\right)$ is its eigenvalue of $i^{\text {th }}$ band. The product is taken for all the occupied bands on the $\Gamma$ point. A simple classification of appearance of DNL is given in Appendix B. An example of 
TABLE I. Character table of the irreducible representations of the little group of the $\Gamma$ point. (1) represents the reality of the representation. A representation with reality (1) has no additional degeneracy under the time-reversal symmetry.

\begin{tabular}{|c|c|c|c|c|c|c|c|c|}
\hline$(\Gamma$ point $)$ & $\{E \mid 000\}$ & $\left\{C_{2 z} \mid 000\right\}$ & $\left\{C_{2 y} \mid \frac{1}{2} \frac{1}{2} 0\right\}$ & $\left\{C_{2 x} \mid \frac{1}{2} \frac{1}{2} 0\right\}$ & $\{I \mid 000\}$ & $\left\{\sigma_{z} \mid 000\right\}$ & $\left\{\sigma_{y} \mid \frac{1}{2} \frac{1}{2} 0\right\}$ & $\left\{\sigma_{x} \mid \frac{1}{2} \frac{1}{2} 0\right\}$ \\
\hline$\Gamma_{1}^{+}(1)$ & 1 & 1 & 1 & 1 & 1 & 1 & 1 & 1 \\
\hline$\Gamma_{1}^{-}(1)$ & 1 & 1 & 1 & 1 & -1 & -1 & -1 & -1 \\
\hline$\Gamma_{2}^{-}(1)$ & 1 & 1 & -1 & -1 & -1 & -1 & 1 & 1 \\
\hline$\Gamma_{3}^{+}(1)$ & 1 & -1 & -1 & 1 & 1 & -1 & -1 & 1 \\
\hline$\Gamma_{3}^{-}(1)$ & 1 & -1 & -1 & 1 & -1 & 1 & 1 & -1 \\
\hline$\Gamma_{4}^{-}(1)$ & 1 & -1 & 1 & -1 & -1 & 1 & -1 & 1 \\
\hline
\end{tabular}

the calculation of Eq. (4) is given in Appendix C. One should note that there is a case system that can have DNL even if $\mathbb{Z}_{2}=1$ (Appendix D). In Appendix E, we discuss applications of the $\mathbb{Z}_{2}$ formula to the other materials with the layer group Nos. 44 and 63. It is of note that the present classification is inherently in 2D and it is distinctive from those established in 3D [34]. Furthermore, by focusing on the mirror-reflection symmetry, the topological invariant, defined in formula (4), clearly distinguishes existence of the nodal-line semimetal in contrast to the previous works $[35,36]$.

\section{B. DFT band calculation}

To confirm the picture, first-principles total-energy calculations in the present research were performed within the framework of density functional theory (DFT) [37,38] as implemented in the QUANTUM ESPRESSO code [39] with the Perdew, Burke, and Ernzerhof exchange-correlation functional [40]. Ultrasoft pseudopotentials were used to describe the electron-ion interaction [41]. Valence wave functions and augmented charge density were expanded using a planewave basis set with cutoff energies of 60 and 480 Ry, respectively. Both the internal atomic coordinates and lattice parameters of borophane (HB) sheet were fully optimized until the residual forces acting on each atom were less than $10^{-4}$ Ry/Bohr. The Brillouin zone integration was sampled by the Monkhorst-Pack (MP) scheme [42] with $8 \times 16 \times 1$ and $8 \times 10 \times 1 k$-point grids in the self-consistent field calculations for optimization structures and energy band structures of (5-7)- $\alpha-\mathrm{B}_{16} \mathrm{H}_{16}$ and (5-6-7)- $\gamma-\mathrm{B}_{24} \mathrm{H}_{24}$ monolayer, respectively. To simulate the isolated borophane (HB) monolayers, the sheet was separated by periodic images with a vacuum region of $15 \AA$. The effect of spin-orbit coupling (SOC) on the electronic structure of the borophane layers was also checked in the DFT calculation. The SOC breaks the Dirac points, as consistent to the other 2D DNL layer [19], and it forms the small energy gap $\leqslant 1 \mathrm{meV}$. The SOC effect on energy bands of all the borophane monolayers found to be negligible, which is expected since both $\mathrm{B}$ and $\mathrm{H}$ are light elements.

Figures 6(a) and 6(c) show the electronic energy band dispersion curves along symmetric axes in the 2D Brillouin zone after the atomic optimization of the (5-7)- $\alpha_{1}-\mathrm{B}_{16} \mathrm{H}_{16}$ and (5-7)- $\alpha_{2}-\mathrm{B}_{16} \mathrm{H}_{16}$ sheets, respectively. In both cases, one can recognize the band crossings or Dirac points (DP) along the $\Gamma-\mathrm{X}, \Gamma-\mathrm{Y}$, and $\Gamma-\mathrm{S}$ lines, indicating the existence of a loop of DPs or DNL. Thus $\mathbb{Z}_{2}=-1$.

The $\psi_{1}$ and $\psi_{2}$ bands, crossing each other, at Fermi level have even $(+)$ and odd $(-)$ parities, respectively. Spatial distributions of the wave functions are illustrated in Fig. 6(b) for $\alpha_{1}-\mathrm{B}_{16} \mathrm{H}_{16}$ and in Fig. 6(d) for $\alpha_{2}-\mathrm{B}_{16} \mathrm{H}_{16}$. At the local B-B bonding sites, wave functions of the $\psi_{1}$ band have a character of the $\sigma$ state, while those of the $\psi_{2}$ band have a character of the $\pi$ state. This result reveals an intriguing relationship between the Dirac fermions and the chemical bonding. The Dirac nodal loop appears when the binding energy of the bonding $\pi$ state, $E_{\pi}$, is lower than that of the bonding $\sigma$ state, $E_{\sigma}$, at $\Gamma\left(k_{x}, k_{y}=0\right)$. This indicates that the bonding configuration is special, $E_{\pi}<E_{\sigma}$, when $\mathbb{Z}_{2}=-1$, while it is normal, $E_{\pi}>E_{\sigma}$, when $\mathbb{Z}_{2}=1$. This relationship between the $\mathbb{Z}_{2}$ topological index and the chemical state may help one to design a novel catalytic reaction on monatomic layers.

The DFT energy band results in Fig. 7 show the presence and absence of DNL in the $\gamma_{1}$ and $\gamma_{2}$ models in Fig. 2. Thus it is $\mathbb{Z}_{2}=-1$ for $\gamma_{1}$, while $\mathbb{Z}_{2}=1$ for $\gamma_{2}$. A collection of the band diagrams and the wave function is exhibited in Appendix F.

Table $\mathrm{V}$ summarizes the topology properties and DFT calculation results of the $\mathrm{HB}$ sheets that are hydrogenated from different membered-ring borophene layers. The number of electrons in a unit cell is even for all the cases and, Thus the electronic structure is semimetallic or insulating. It should note that the $\mathrm{B}_{2} \mathrm{H}_{2}$ sheet, which is composed of 6-membered rings of boron atoms, has been experimentally reported [28,29]. The $\mathbb{Z}_{2}$ topological index, evaluated by

TABLE II. Character table of the irreducible representations of the little group of the $X$ and $Y$ points. (1) represents the reality of the representation. A representation with reality (1) has no additional degeneracy under the time-reversal symmetry.

\begin{tabular}{|c|c|c|c|c|c|c|c|c|c|}
\hline$(X$ point $)$ & (Y point $)$ & $\{E \mid 000\}$ & $\left\{C_{2 z} \mid 000\right\}$ & $\left\{C_{2 y} \mid \frac{1}{2} \frac{1}{2} 0\right\}$ & $\left\{C_{2 x} \mid \frac{1}{2} \frac{1}{2} 0\right\}$ & $\{I \mid 000\}$ & $\left\{\sigma_{z} \mid 000\right\}$ & $\left\{\sigma_{y} \mid \frac{1}{2} \frac{1}{2} 0\right\}$ & $\left\{\sigma_{x} \mid \frac{1}{2} \frac{1}{2} 0\right\}$ \\
\hline$X_{1}(1)$ & $Y_{2}(1)$ & 2 & 0 & 0 & 0 & 0 & 2 & 0 & 0 \\
\hline$X_{2}(1)$ & $Y_{1}(1)$ & 2 & 0 & 0 & 0 & 0 & -2 & 0 & 0 \\
\hline
\end{tabular}


TABLE III. Character table of the irreducible representations of the little group of the $C$ point (line) and the $D$ point (line). (0) represents the reality of the representation. A representation with reality (0) degenerate with another different representation under the time-reversal symmetry. The pair that degenerate under the timereversal symmetry is shown in the bottom of the table.

\begin{tabular}{lccccc}
\hline \hline (C point) & & $\{E \mid 000\}$ & $\left\{C_{2 x} \mid \frac{1}{2} \frac{1}{2} 0\right\}$ & $\left\{\sigma_{z} \mid 000\right\}$ & $\left\{\sigma_{y} \mid \frac{1}{2} \frac{1}{2} 0\right\}$ \\
& $(D$ point $)$ & $\{E \mid 000\}$ & $\left\{C_{2 y} \mid \frac{1}{2} \frac{1}{2} 0\right\}$ & $\left\{\sigma_{z} \mid 000\right\}$ & $\left\{\sigma_{x} \mid \frac{1}{2} \frac{1}{2} 0\right\}$ \\
\hline$C_{1}(0)$ & $D_{1}(0)$ & 1 & $e^{-i \pi k}$ & 1 & $e^{-i \pi k}$ \\
$C_{2}(0)$ & $D_{2}(0)$ & 1 & $-e^{-i \pi k}$ & 1 & $-e^{-i \pi k}$ \\
$C_{3}(0)$ & $D_{3}(0)$ & 1 & $e^{-i \pi k}$ & -1 & $-e^{-i \pi k}$ \\
$C_{4}(0)$ & $D_{4}(0)$ & 1 & $-e^{-i \pi k}$ & -1 & $e^{-i \pi k}$ \\
\hline
\end{tabular}

for $C$ point $\boldsymbol{k}_{C}=\left(k, \frac{1}{2}, 0\right),\left(C_{1}, C_{2}\right),\left(C_{3}, C_{4}\right)$

for $D$ point $\boldsymbol{k}_{D}=\left(\frac{1}{2}, k, 0\right),\left(D_{1}, D_{2}\right),\left(D_{3}, D_{4}\right)$

Eq. (4), can be defined for layers of $\alpha_{1^{-}}, \alpha_{2^{-}}, \gamma_{1^{-}}$, and $\gamma_{2^{-}}$ borophane that belong to the layer group No. 44. Based on the DFT calculations, we find that the $\alpha_{1^{-}}, \alpha_{2^{-}}$, and $\gamma_{1}$-borophane sheet are semimetals with DNL, while the $\gamma_{2}$-borophane is an insulator. The $\gamma_{3^{-}}, \gamma_{4^{-}}$, and $\mathrm{B}_{2} \mathrm{H}_{2}$ borophane are semimetals without DNL. The layers of DNL semimetal or insulator is consistent with the $\mathbb{Z}_{2}$ topological index. It is noted that a recent report [36] has established the $\mathbb{Z}_{2}$ topological index for borophene or borophane models. This topological taxonomy helps to classify semimetallic layers with Dirac points or Dirac nodal loops, while our approach of the $\mathbb{Z}_{2}$ index distinguishes between layers of the Dirac nodal semimetal and those of insulator.

\section{Natural existence of the borophane}

\section{Cohesive energy}

Finally, it is important to consider the experimental realization of the borophane sheets. The values of cohesive energy $\left(E_{C}\right)$ of the $\mathrm{HB}$ sheets are listed in Table V. The energy is defined as $E_{C}=\left(n_{\mathrm{B}} E_{\mathrm{B}}+n_{\mathrm{H}} E_{\mathrm{H}}-E_{\mathrm{BH}}\right) /\left(n_{\mathrm{B}}+n_{\mathrm{H}}\right)$, where $n_{\mathrm{B}}\left(n_{\mathrm{H}}\right)$ and $E_{\mathrm{B}}\left(E_{\mathrm{H}}\right)$ are a number and energy of boron (hydrogen) atom, respectively, while $E_{B H}$ is the total energy of a $\mathrm{HB}$ sheet. The calculation indicates the $E_{C}$ values for the (5-7)- $\alpha-\mathrm{B}_{16} \mathrm{H}_{16}$, and (5-7)- $\gamma-\mathrm{B}_{24} \mathrm{H}_{24}$ sheets are marginally smaller than that of the synthesized $\mathrm{B}_{2} \mathrm{H}_{2}$
TABLE V. Optimized lattice constants and polygons in the membered rings of the individual HB sheets are listed. The $\mathbb{Z}_{2}$ topological indexes are calculated by Eq. (1) for layers belong to the layer group No. 44. The existence of Dirac nodal loops is confirmed by the DFT calculation. Values of cohesive energy $E_{C}$ are quantified by the DFT calculations.

\begin{tabular}{lcccccc}
\hline \hline HB sheet & $\begin{array}{c}a \\
(\AA)\end{array}$ & $\begin{array}{c}b \\
(\AA)\end{array}$ & $\begin{array}{c}\text { Membered } \\
\text { rings }\end{array}$ & $\begin{array}{c}\mathbb{Z}_{2} \\
\text { index }\end{array}$ & $\begin{array}{c}\text { Dirac nodal } \\
\text { loop }\end{array}$ & $\begin{array}{c}E_{C} \\
(\mathrm{eV} / \text { atom })\end{array}$ \\
\hline $\mathrm{B}_{2} \mathrm{H}_{2}$ & 5.287 & 3.018 & 6 & & No & 4.08 \\
$\alpha_{1}-\mathrm{B}_{16} \mathrm{H}_{16}$ & 11.292 & 5.875 & 5,7 & -1 & Yes & 4.02 \\
$\alpha_{2}-\mathrm{B}_{16} \mathrm{H}_{16}$ & 11.301 & 5.829 & 5,7 & -1 & Yes & 4.04 \\
$\gamma_{1}-\mathrm{B}_{24} \mathrm{H}_{24}$ & 11.067 & 8.871 & $5,6,7$ & -1 & Yes & 4.05 \\
$\gamma_{2}-\mathrm{B}_{24} \mathrm{H}_{24}$ & 11.125 & 8.867 & $5,6,7$ & 1 & No & 4.04 \\
$\gamma_{3}-\mathrm{B}_{24} \mathrm{H}_{24}$ & 11.110 & 8.820 & $5,6,7$ & & No & 4.05 \\
$\gamma_{4}-\mathrm{B}_{24} \mathrm{H}_{24}$ & 11.146 & 8.809 & $5,6,7$ & & No & 4.05 \\
\hline \hline
\end{tabular}

borophane sheet [28,29] about 30-50 meV. These differences in energy values are comparable to the thermal energy $k_{B} T$ at room temperature, indicating the possible existence of the $\alpha$ and $\gamma$-HB sheets.

\section{Lattice dynamic and thermal stability}

The calculations of the phonon dispersion with Phonopy code [43] and first-principles molecular dynamics (FPMD) simulations were performed to reveal thermal stability and lattice dynamics for the (5-7)- $\alpha-\mathrm{B}_{16} \mathrm{H}_{16}$ and (5-6-7)- $\gamma_{1}-\mathrm{B}_{24} \mathrm{H}_{24}$ monolayers. In the calculation of the phonon dispersion, GGA-PBE [40] was adopted for a pseudopotential and interlayer spacing between the sheets was $15 \AA$. In the FPMD simulation with the canonical (NVT) ensemble, the simulations were performed with a Berendsen thermostat. In both calculations, the $2 \times 2 \times 1$ supercells were constructed.

Figure 8 shows phonon dispersion diagrams for layers of (5-7)- $\alpha_{1}-\mathrm{B}_{16} \mathrm{H}_{16},(5-7)-\alpha_{2}-\mathrm{B}_{16} \mathrm{H}_{16}$, and (5-6-7)- $\gamma_{1}-\mathrm{B}_{24} \mathrm{H}_{24}$ that have the Dirac nodal Fermions. All the dispersion curves of (5-7)- $\alpha_{1}-\mathrm{B}_{16} \mathrm{H}_{16}$ and (5-7)- $\alpha_{2}-\mathrm{B}_{16} \mathrm{H}_{16}$ have positive frequency, while some curves of (5-6-7)- $\gamma_{1}-\mathrm{B}_{24} \mathrm{H}_{24}$ are negative. This indicates stability of (5-7)- $\alpha-\mathrm{B}_{16} \mathrm{H}_{16}$ layers, while it is unlikely that there is a layer of (5-6-7)- $\gamma_{1}-\mathrm{B}_{24} \mathrm{H}_{24}$ in nature.

The existence of the (5-7)- $\alpha_{1}-\mathrm{B}_{16} \mathrm{H}_{16}$ layer is further examined by simulating lattice dynamics at $700 \mathrm{~K}$. As

TABLE IV. Character table of the irreducible representations of the little group of the $S$ point. (0) represents the reality of the representation. A representation with reality $(0)$ degenerate with another different representation under the time-reversal symmetry. The pair that degenerate under the time-reversal symmetry is shown in the bottom of the table.

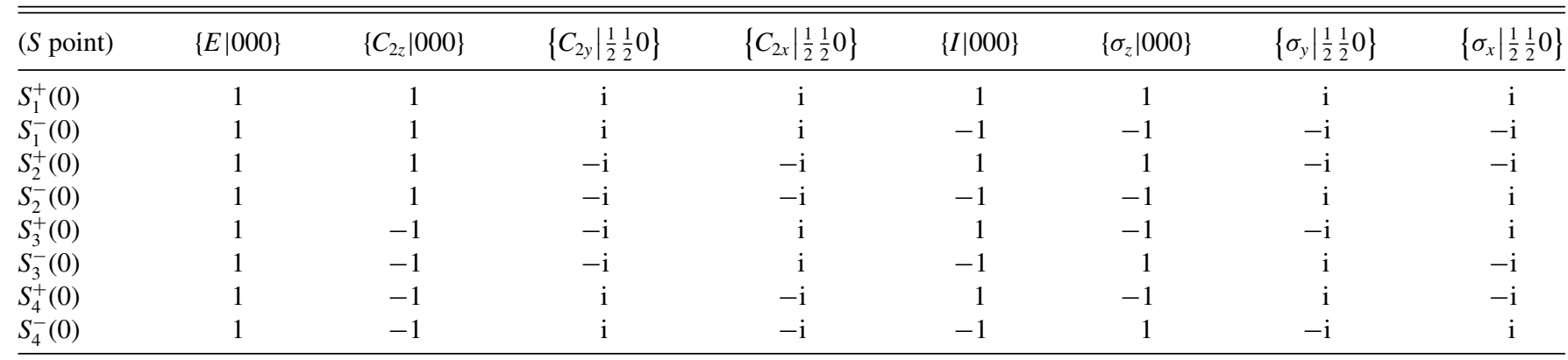

$\left(S_{1}^{+}, S_{2}^{+}\right),\left(S_{1}^{-}, S_{2}^{-}\right),\left(S_{3}^{+}, S_{4}^{+}\right),\left(S_{3}^{-}, S_{4}^{-}\right)$ 


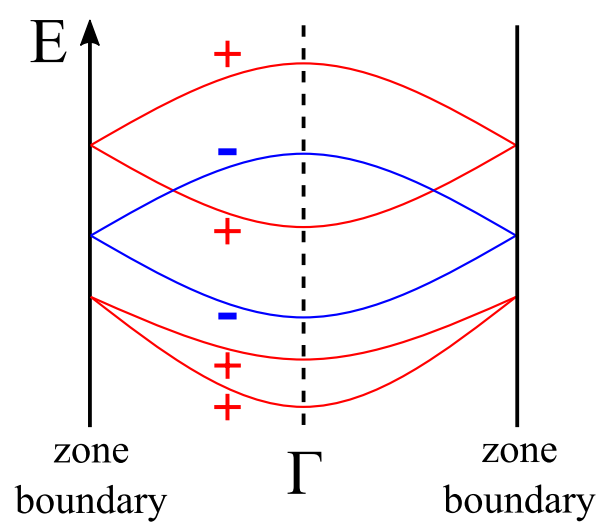

FIG. 5. Schematic picture of the band dispersion in the layer group No. 44. All bands on the zone boundary are doubly degenerate and the degenerate pairs have the same eigenvalues.

shown in Fig. 9, one can confirm that atomic structure of (5-7)- $\alpha_{1}-\mathrm{B}_{16} \mathrm{H}_{16}$ maintains after $5 \mathrm{ps}$. The simulation result for (5-7)- $\alpha_{2}-\mathrm{B}_{16} \mathrm{H}_{16}$ also shows the conservation of the atomic structure at $700 \mathrm{~K}$ (Fig. 10). These results indicate that layers of (5-7)- $\alpha-\mathrm{B}_{16} \mathrm{H}_{16}$ are stable.

Our results of phonon dispersion and molecular dynamics simulations indicate the lattice dynamics and thermal stability of the (5-7)- $\alpha-\mathrm{B}_{16} \mathrm{H}_{16}$ monolayers. Thus one can expect the free-standing (5-7)- $\alpha-\mathrm{B}_{16} \mathrm{H}_{16}$ sheets to be the Dirac nodal semimetal. It is worth mentioning that, in previous works $[28,29]$, we synthesized the $\mathrm{B}_{2} \mathrm{H}_{2}$ sheets by the proton ion-exchange reaction of $\mathrm{MgB}_{2}$ and liquid exfoliation. By the same chemical process, layers of $\alpha-\mathrm{B}_{16} \mathrm{H}_{16}$ may be made from crystals of the $\mathrm{TmAlB}_{4}$ such as $\mathrm{YCrB}_{4}$.
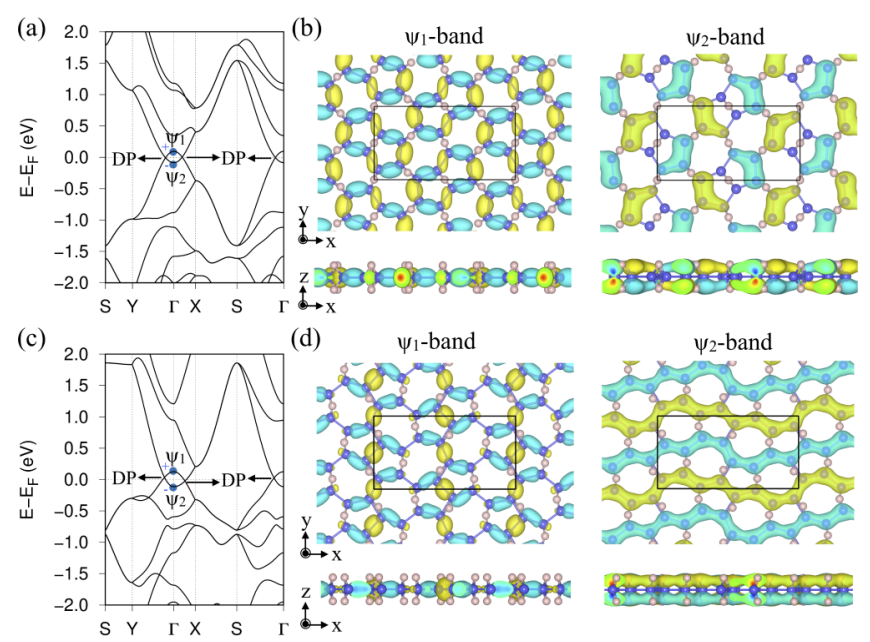

FIG. 6. Electronic energy band dispersions [(a) and (c)] along the $k$ path in the Brillouin zone and spatial distributions of wave functions [(b) and (d)] of $\psi_{1}$ and $\psi_{2}$ bands at the $\Gamma$ point for (5-7)- $\alpha_{1}-\mathrm{B}_{16} \mathrm{H}_{16}$ and (5-7)- $\alpha_{2}-\mathrm{B}_{16} \mathrm{H}_{16}$, respectively. $E_{F}$ is the Fermi energy. DP means Dirac point. The color of the wave functions corresponds to the sign of wave functions. The parity of mirror reflection symmetry with respect to the $x-y$ plane (layer plane) for $\psi_{1}$ and $\psi_{2}$ bands are labeled with + and - signs, respectively.
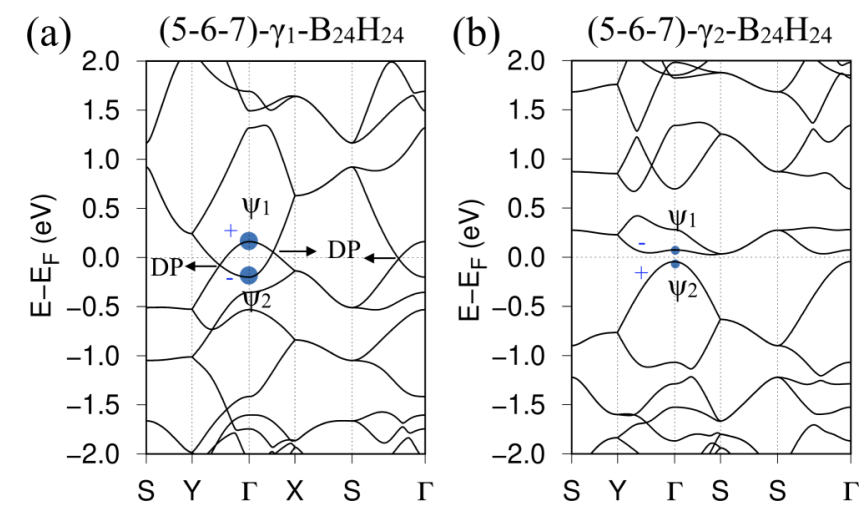

FIG. 7. Energy band dispersion of (a) (5-6-7)- $\gamma_{1}-\mathrm{B}_{24} \mathrm{H}_{24}$ and (b) (5-6-7)- $\gamma_{2}-\mathrm{B}_{24} \mathrm{H}_{24}$. The parity of mirror reflection symmetry with respect to the $x$ - $y$ plane (layer plane) for $\psi_{1}$ and $\psi_{2}$ bands are labeled with + and - signs, respectively. Two-dimensional Brillouin zone are shown with symbols at the symmetry points.

\section{SUMMARY}

In summary, we presented the prediction of 2D DNLs in nonsymmorphic symmetry borophane (HB) sheets. The existence of DNL was evaluated by the $\mathbb{Z}_{2}$ topological index for several borophane layers and confirmed by the first-principles DFT calculations. The present taxonomy of topology can be applied to design novel atomic sheets with DNL fermions. Moreover, the calculations suggest the existence of $\alpha-\mathrm{B}_{16} \mathrm{H}_{16}$ that may be synthesized from a $\mathrm{TmAlB}_{4}$-type crystal. Our results suggest that the $\mathrm{HB}$ sheet is a promising $2 \mathrm{D}$ material for realizing novel functional devices.

\section{ACKNOWLEDGMENTS}

This work was supported by a Grant-in-Aid for Specially Promoted Research (KAKENHI 18H03874, 19H04398) from the Japan Society for the Promotion of Science (JSPS) and JST-CREST Grant No. JPMJCR1715 from the Japan Science and Technology Agency. M.O. was supported by KAKENHI $18 \mathrm{H} 01162$ from JSPS. K.Y. was supported by KAKENHI 19K05643 from JSPS. I.T. was supported from JSPS by KAKENHI 17H02912 and through the Program for Leading Graduate Schools (MERIT). Yuki Tsujikawa and Yusuke Sato were acknowledged for discussion. A part of the simulations was performed on the SGI ICE XA/UV supercomputer at the Institute of Solid State Physics, The University of Tokyo. T.K. was supported by MEXT Element Strategy Initiative to Form Core Research Center (JPMXP0112101001). T.K. and B.S.
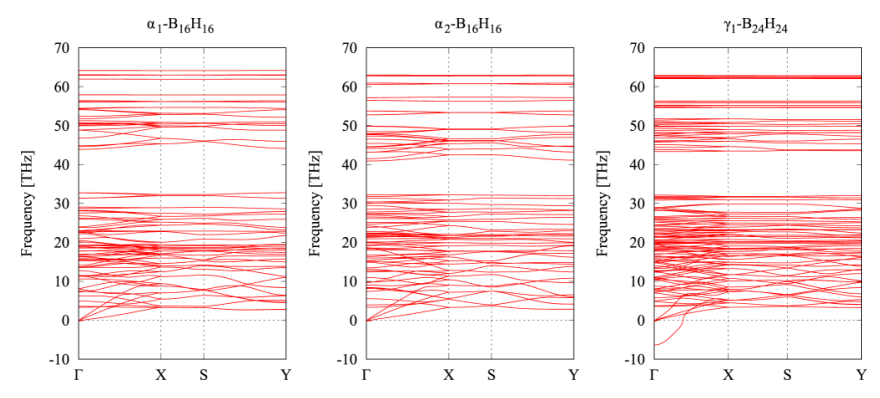

FIG. 8. Phonon dispersion diagrams of layers of (5-7)- $\alpha_{1}-\mathrm{B}_{16} \mathrm{H}_{16},(5-7)-\alpha_{2}-\mathrm{B}_{16} \mathrm{H}_{16}$, and (5-6-7)- $\gamma_{1}-\mathrm{B}_{24} \mathrm{H}_{24}$. 



FIG. 9. Simulation of atomic structure of the (5-7)- $\alpha_{1}-\mathrm{B}_{16} \mathrm{H}_{16}$ after 5.0 ps at $700 \mathrm{~K}$.

were supported by JSPS Core-to-Core Program. B.S. was supported by EPSRC Grant No. EP/R034540/1 Defect Functionalized Sustainable Energy Materials: From Design to Devices.

\section{APPENDIX A: A NUMBER OF ELECTRONS IN A UNIT CELL}

The ratio of hydrogen and boron in a unit cell is $\mathrm{H}: \mathrm{B}=1: 1$. Additionally, the layer group No. 44 has nonsymmorphic operations, $C_{2}$ screws and glides, and it requires the number of each atom in a unit cell to be an even number. When the layer group symmetry is given and an atom is placed in the unit cell, some other atoms must be placed in the equivalent position in the unit cell to satisfy the layer group symmetry. Especially for the layer group No. 44, the set of atoms in the equivalent position must contain an even number of atoms. In other words, all Wyckoff positions have an even number of multiplicity [44]. For example, the case of $\alpha$-borophene is shown in Fig. 11. The red point in Fig. 11 in the corner (origin $(0,0,0))$ and the center $\left[\left(\frac{1}{2}, \frac{1}{2}, 0\right)\right]$ in the unit cell are equivalent. There are two red points per unit cell. For boron atoms in Fig. 11, there are four equivalent boron atoms per unit cell (equivalent atoms are marked with the same symbol.). The red point is placed in the Wyckoff position $2 a$ and boron atoms are placed in the Wyckoff position $4 g$, where the notation of the Wyckoff position of the 3D space group No. 55 is used. From these facts, the chemical structural formula of $\mathrm{HB}$ sheets is written as $\mathrm{B}_{2 n} \mathrm{H}_{2 n}$. Therefore the number of the electron in a unit cell (filling factor) is $12 n=4 n^{\prime}\left(n, n^{\prime} \in \mathbb{N}\right)$.

\section{APPENDIX B: EXISTENCE OF DIRAC NODAL LOOP (DNL)}

Electronic wave functions of $2 \mathrm{D}$ crystal materials in this layer group No. 44 have even and odd parities by the mirror


FIG. 10. Simulation of atomic structure of the (5-7)- $\alpha_{2}-\mathrm{B}_{16} \mathrm{H}_{16}$ after 5.0 ps at $700 \mathrm{~K}$.

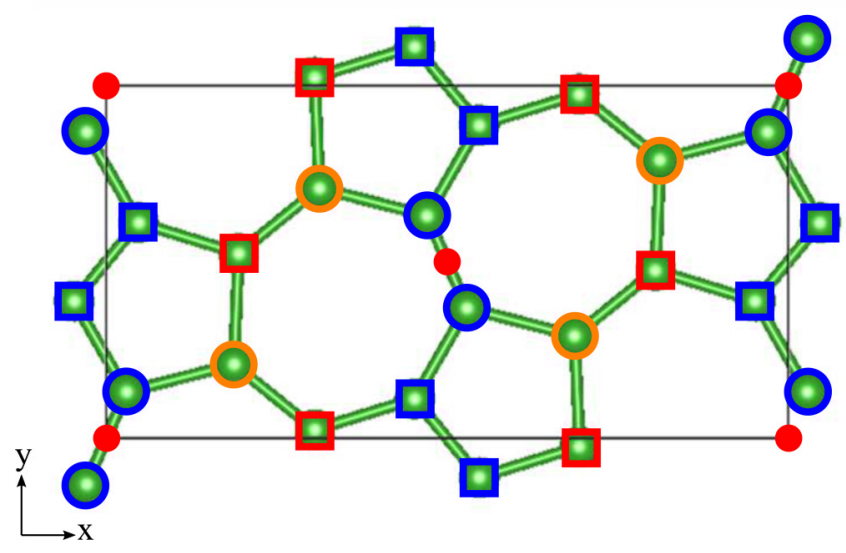

FIG. 11. Structure of $\alpha$-borophene as an example of the layer group No. 44. The equivalent points atoms are indicated with same symbols (red point, blue circle, orange circle, red square and blue square). When a bond is placed at the corner of the unit cell (red point), another bond must be placed at the center of the unit cell (shown with another red point) to satisfy the symmetry of the layer group No. 44 . When an atom is placed at the position indicated with blue circle, other three atoms must be placed at the position indicated with other three blue circles.

symmetry for the $x-y$ plane (layer plane). It has been proved that these doubly degenerate bands have the same parities, as shown in Fig. 12. Thus, when one takes a product of the parities of the wave functions of occupied bands, it is even along all the zone boundaries, including the $X, Y$, and $S$ points as well as the symmetry lines along $X-S-Y$ [32]. On the other hand, there is no restricted degeneracy on the $\Gamma$ point and a product is even or odd at the $\Gamma$ point. When the product of parities on the $\Gamma$ point is even, there is a band gap [Fig. 12(a)]. When it is odd, as shown in Fig. 12(b), the number of bands with odd parities is an odd number on the $\Gamma$ point and there must be a crossing of bands with different parities between (a)

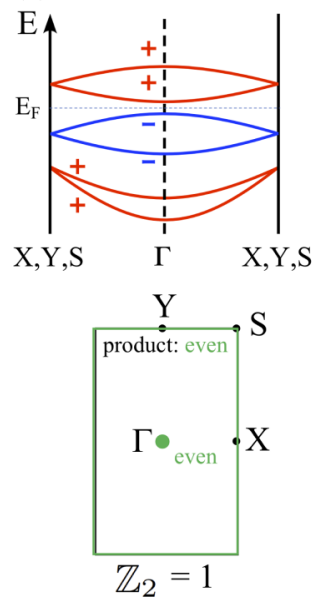

(b)

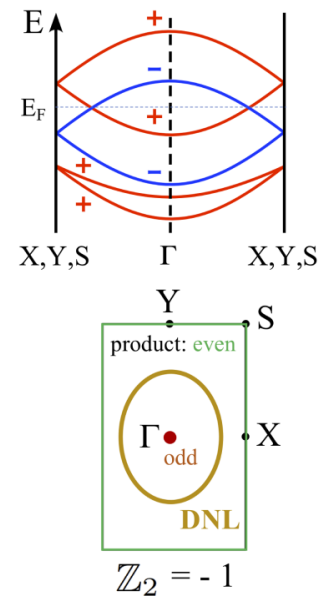

(c)

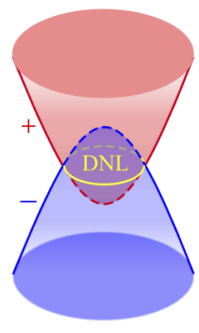

FIG. 12. Schematic picture of the band dispersion in the layer group No. 44. Electronic bands of odd and even symmetries are labeled + and - , respectively. A relation between a DNL and the symmetry points of the Brillouin zone for the cases (a) without and (b) with DNL. (c) A schematic drawing of DNL. 
TABLE VI. Irreducible representations (irrep) of the bands in the $\Gamma$ point and its $\left\{\sigma_{z} \mid 000\right\}$ eigenvalues for the (5-7)- $\alpha_{2}-\mathrm{B}_{16} \mathrm{H}_{16}$ borophane sheet. The irreps are written with the notation we used in Table I. $E_{f}$ means Fermi energy and thus there are 32 occupied bands.

\begin{tabular}{lcccccccccc}
\hline \hline Number of band & 1 & 2 & 3 & 4 & 5 & 6 & 7 & 8 & 9 & 10 \\
\hline irrep & $\Gamma_{1}^{+}$ & $\Gamma_{2}^{+}$ & $\Gamma_{4}^{-}$ & $\Gamma_{3}^{-}$ & $\Gamma_{4}^{-}$ & $\Gamma_{1}^{+}$ & $\Gamma_{3}^{-}$ & $\Gamma_{2}^{+}$ & $\Gamma_{2}^{-}$ & $\Gamma_{1}^{-}$ \\
$\left\{\sigma_{z} \mid 000\right\}$ eigenvalue & + & + & + & + & + & + & + & + & - & - \\
Number of band & 11 & 12 & 13 & 14 & 15 & 16 & 17 & 18 & 19 & 20 \\
\hline irrep & $\Gamma_{2}^{+}$ & $\Gamma_{1}^{+}$ & $\Gamma_{3}^{+}$ & $\Gamma_{3}^{-}$ & $\Gamma_{4}^{+}$ & $\Gamma_{2}^{-}$ & $\Gamma_{3}^{+}$ & $\Gamma_{2}^{+}$ & $\Gamma_{3}^{-}$ & $\Gamma_{4}^{+}$ \\
$\left\{\sigma_{z} \mid 000\right\}$ eigenvalue & + & + & - & + & - & - & - & + & + & - \\
Number of band & 21 & 22 & 23 & 24 & 25 & 26 & 27 & 28 & 29 & 30 \\
\hline irrep & $\Gamma_{1}^{-}$ & $\Gamma_{1}^{+}$ & $\Gamma_{4}^{-}$ & $\Gamma_{4}^{-}$ & $\Gamma_{2}^{+}$ & $\Gamma_{4}^{-}$ & $\Gamma_{1}^{+}$ & $\Gamma_{1}^{+}$ & $\Gamma_{2}^{+}$ & $\Gamma_{2}^{+}$ \\
$\left\{\sigma_{z} \mid 000\right\}$ eigenvalue & - & + & + & + & + & + & + & + & + & + \\
Number of band & 31 & 32 & $\left(E_{f}\right)$ & 33 & 34 & 35 & & & & \\
\hline irrep & $\Gamma_{3}^{-}$ & $\Gamma_{1}^{-}$ & \multicolumn{1}{c}{$\Gamma_{1}^{+}$} & $\Gamma_{2}^{-}$ & $\Gamma_{4}^{+}$ & & & & \\
$\left\{\sigma_{z} \mid 000\right\}$ eigenvalue & + & - & \multicolumn{1}{c}{+} & - & - & & & & \\
\hline \hline
\end{tabular}

the $\Gamma$ point and the zone boundary. In this case, one expects the existence of the Dirac nodal loop (DNL), as drawn in Fig. 12(c).

\section{APPENDIX C: AN EXAMPLE OF THE CALCULATION OF OUR $\mathbb{Z}_{2}$ FORMULA}

We show an example of the calculation of our $\mathbb{Z}_{2}$ formula by using the band dispersion of the (5-7)- $\alpha_{2}-\mathrm{B}_{16} \mathrm{H}_{16}$ borophane sheet. For each band in the $\Gamma$ point, the irreducible representation and its eigenvalue of $\left\{\sigma_{z} \mid 000\right\}$ operation are listed in Table VI. The number of the band is defined from the lowest energy band. In this calculation, the electrons from 1s orbital of Boron atoms are neglected as inner shell electrons. The Fermi energy lies between the 32nd band and the 33rd band. These irreducible representations are given by QUANTUM ESPRESSO [39]. Although there are some choices for the origin of symmetry operation, it doesn't matter in the eigenvalue of the symmetry operation in the $\Gamma$ point. By seeing the Table VI, we can calculate the $\mathbb{Z}_{2}$ number as

$$
\mathbb{Z}_{2}=\prod_{\text {occupied }} \Gamma_{i}\left(\sigma_{z}\right)=(+1)^{23}(-1)^{9}=-1 .
$$

\section{APPENDIX D: A CASE OF DNL WITH $\mathbb{Z}_{2}=1$}

We note that even if $\mathbb{Z}_{2}=1$, the system can have Dirac nodal loops. For example, the cases where two bands are inverted [Fig. 13(a)] and where the band inversion occurs only some part of the $\Gamma$-boundary line [Fig. 13(b)] have Dirac nodal loops but $\mathbb{Z}_{2}=1$. However, in these cases, the number of the Dirac nodal loops must be an even number. For the system with an odd number of Dirac nodal loops, the $\mathbb{Z}_{2}$ number is always nontrivial. More precise analysis, for example explicitly counting the number of bands with $+1(-1)$ eigenvalues, can distinguish the Fig. 13(a) system and the system without Dirac nodal loops. Nevertheless, it

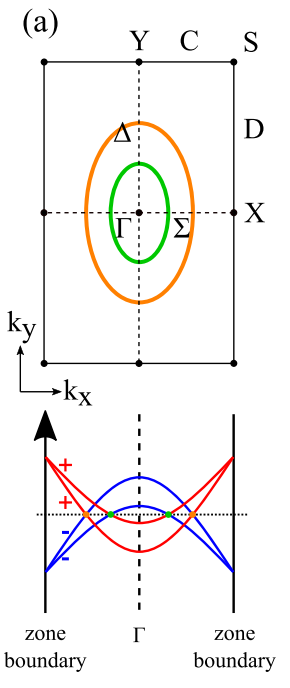

(b)
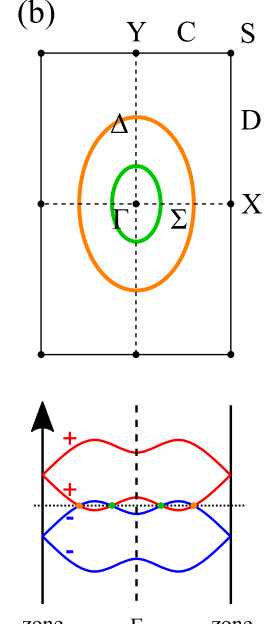

zone

boundary boundary (c)
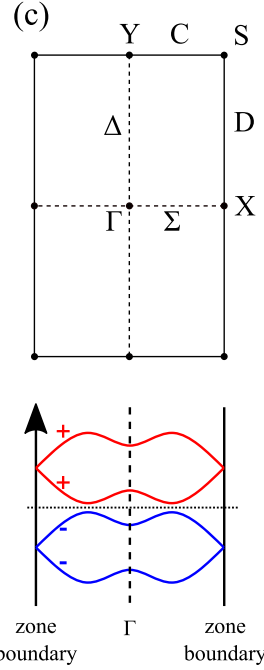

FIG. 13. Schematic pictures of Brillouin zone, nodal lines and band dispersion for the system with [(a) and (b)] two nodal lines and $\mathbb{Z}_{2}=0$ and (c) no nodal line and $\mathbb{Z}_{2}=0$. (a) Two bands are inverted and as a result the $\mathbb{Z}_{2}$ is kept trivial. (b) The band inversion occurs only on some part of the line between the $\Gamma$ point and the zone boundary. In this case, the symmetry of wave function on the $\Gamma$ point is not changed by the band inversion. Thus it is essentially impossible to distinguished (b) and (c) by examining the $\Gamma$ point.

is essentially impossible to distinguish Figs. 13(b) and 13(c) only by examining the high-symmetry point because their band inversion makes no difference on the high-symmetry point.

\section{APPENDIX E: OTHER APPLICATIONS}

We can also apply this $\mathbb{Z}_{2}$ formula to other materials with the layer group Nos. 44 and 63 in addition to HB sheets. For example, transition metal dichalcogenide haeckelites [45] has the symmetry of the layer group No. 44 and the ShastrySutherland lattice has the symmetry of the layer group No. 63. For haeckelite, the Dirac points can appear. By combining the $\mathbb{Z}_{2}$ formula, we proposed above and previous $\mathbb{Z}_{2}$ index $v_{3}$ [46] defined as

$$
(-1)^{\nu_{3}}=\prod_{\text {occupied, 4TRIM }} \Gamma_{i}(I)
$$

TABLE VII. Topological numbers and the system that is realized in each phase. $\mathbb{Z}_{2}$ means the $\mathbb{Z}_{2}$ formula we defined in this work and $\nu_{3}$ is the previous $\mathbb{Z}_{2}$ index defined in a previous study [46]. When $\mathbb{Z}_{2}=1$, the system must have a Dirac nodal loop. When $\mathbb{Z}_{2}=0$ and $v_{3}=1$, the system does not have Dirac nodal loop but must have Dirac node points. When $\mathbb{Z}_{2}=0$ and $\mathbb{Z}_{2}=0$, the system can have an even number of Dirac nodal loops, Dirac node points or nothing (normal insulator).

\begin{tabular}{lcc}
\hline \hline & $\mathbb{Z}_{2}=1$ & $\mathbb{Z}_{2}=-1$ \\
\hline$\nu_{3}=0$ & & Dirac nodal loop \\
$\nu_{3}=1$ & Dirac point & Dirac nodal loop \\
\hline \hline
\end{tabular}


(a)



(c)

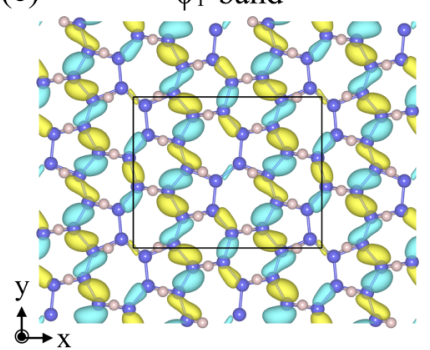

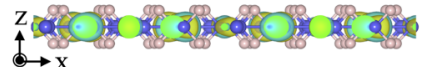

(b)

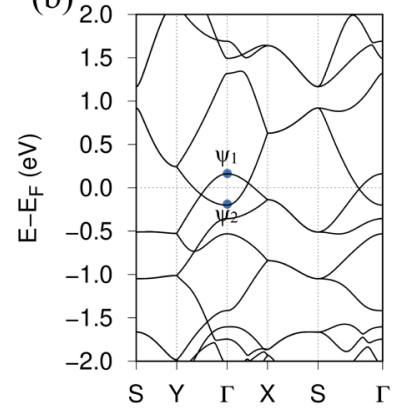

(d)
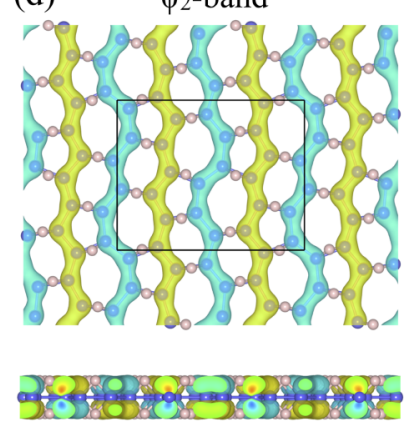

FIG. 14. (a) Atomic structure and (b) energy band dispersion of (5-6-7)- $\gamma_{1}-\mathrm{B}_{24} \mathrm{H}_{24}$. [(c) and (d)] Spatial distributions of wave functions of $\psi_{1}$ and $\psi_{2}$ bands at the $\Gamma$ point. Locations of the 5-membered and 6-membered rings are indicated with red pentagons and purple hexagons, respectively. $E_{F}$ is the Fermi energy. The color of wave functions corresponds to the sign of wave functions.

(a)

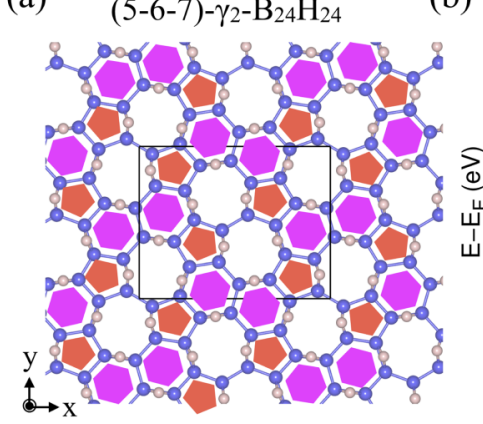

(c)

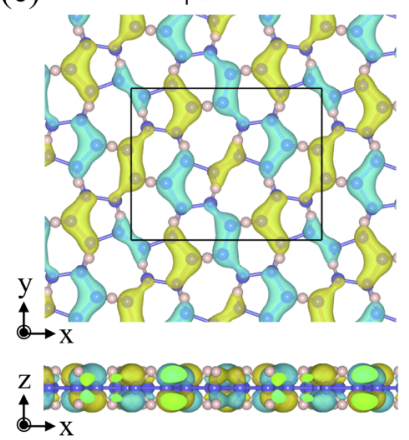

(b)

(d)
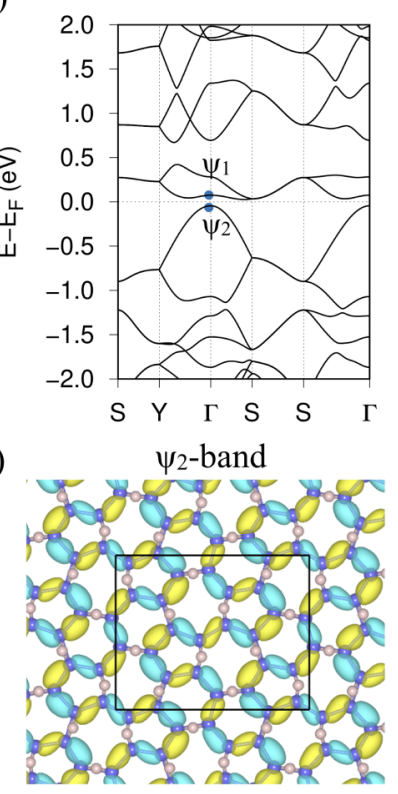

18.

FIG. 15. (a) Atomic structure and (b) energy band dispersion of (5-6-7)- $\gamma_{2}-\mathrm{B}_{24} \mathrm{H}_{24}$. [(c) and (d)] Spatial distributions of wave functions of $\psi_{1}$ and $\psi_{2}$ bands at the $\Gamma$ point. Locations of the 5-membered and 6-membered rings are indicated with red pentagons and purple hexagons, respectively. $E_{F}$ is the Fermi energy. The color of wave functions corresponds to the sign of wave functions.

(a) (5-6-7)- $\gamma_{3}-\mathrm{B}_{24} \mathrm{H}_{24}$



(c)

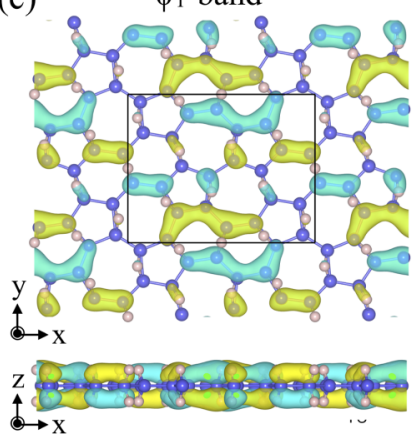

(b)



(d)

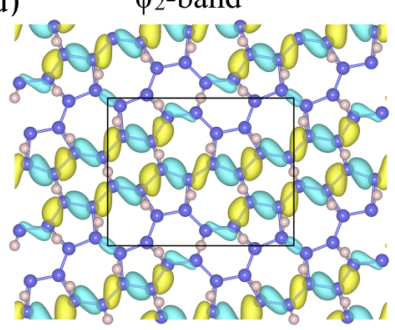

2.80
FIG. 16. (a) Atomic structure and (b) energy band dispersion of (5-6-7)- $\gamma_{3}-\mathrm{B}_{24} \mathrm{H}_{24}$. [(c) and (d)] Spatial distributions of wave functions of $\psi_{1}$ and $\psi_{2}$ bands at the $\Gamma$ point. Locations of the 5-membered and 6-membered rings are indicated with red pentagons and purple hexagons, respectively. $E_{F}$ is the Fermi energy. The color of wave functions corresponds to the sign of wave functions. (a)

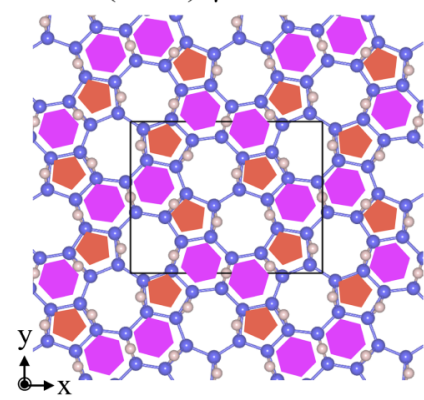

(c)

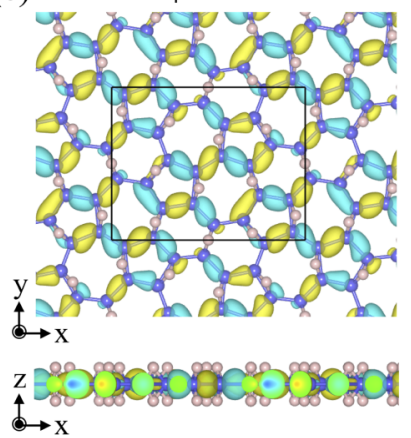

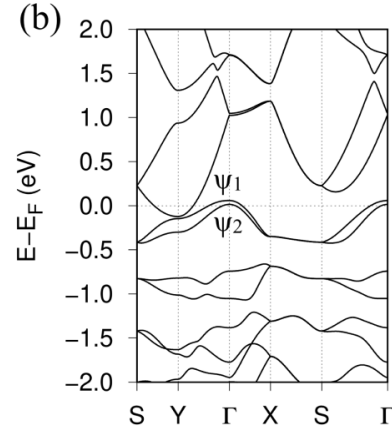

(d)



FIG. 17. (a) Atomic structure and (b) energy band dispersion of (5-6-7)- $\gamma_{4}-\mathrm{B}_{24} \mathrm{H}_{24}$. [(c) and (d)] Spatial distributions of wave functions of $\psi_{1}$ and $\psi_{2}$ bands at the $\Gamma$ point. Locations of the 5-membered and 6-membered rings are indicated with red pentagons and purple hexagons, respectively. $E_{F}$ is the Fermi energy. The color of wave functions corresponds to the sign of wave functions. 
we can diagnose whether the system has a Dirac nodal loop or a Dirac point (Table VII). The $v_{3}$ is originally defined to diagnose the existence of the Dirac nodal line in the 3D Brillouin zone. Although the $v_{3}$ can be calculated for a 2D system $\left(k_{z}=0\right)$, the $v_{3}$ represents how many nodal loops in 3D Brillouin zone which is given when the $c$ axis (along the $z$ direction) is assumed to be finite. If the nodal loop predicted by $v_{3}=1$ is perpendicular to the $k_{z}=0$ plane, the degeneracy will be recognized as point nodes in the original 2D Brillouin zone $\left(k_{x}-k_{y}\right.$ space). Some of the transition metaldichalcogenide haeckelites have degenerate points on its high-symmetry lines. However, in those systems, $\mathbb{Z}_{2}=0$ and the degenerate point is the Dirac point (not Dirac nodal loop).

\section{APPENDIX F: A COLLECTION OF DFT RESULTS ON $\gamma$-BOROPHANE}

Figures 14-17 show atomic structures and electronic states of the (5-6-7)- $\gamma$-borophane of the four different bonding configurations, $\gamma_{1^{-}}, \gamma_{2-}, \gamma_{3}-$, and $\gamma_{4}-\mathrm{B}_{24} \mathrm{H}_{24}$, respectively.
[1] K. S. Novoselov, A. Mishchenko, A. Carvalho, and A. H. C. Neto, Science 353, aac9439 (2016).

[2] A. D. Franklin, Science 349, aab2750 (2015).

[3] I. Matsuda (ed.), Monatomic Two-Dimensional Layers: Modern Experimental Approaches for Structure, Properties, and Industrial Use (Elsevier, Amsterdam, 2018).

[4] P. Vogt, P. DePadova, C. Quaresima, J. Avila, E. Frantzeskakis, M. C. Asensio, A. Resta, B. Ealet, and G. Le Lay, Phys. Rev. Lett. 108, 155501 (2012).

[5] A. Fleurence, R. Friedlein, T. Ozaki, H. Kawai, Y. Wang, and Y. Yamada-Takamura, Phys. Rev. Lett. 108, 245501 (2012).

[6] C.-L. Lin, R. Arafune, K. Kawahara, N. Tsukahara, E. Minamitani, Y. Kim, N. Takagi, and M. Kawai, Appl. Phys. Express 5, 045802 (2012).

[7] B. Feng, H. Zhou, Y. Feng, H. Liu, S. He, I. Matsuda, L. Chen, E. F. Schwier, K. Shimada, S. Meng, and K. Wu, Phys. Rev. Lett. 122, 196801 (2019).

[8] M. E. Davila, L. Xian, S. Cahangirov, A. Rubio, and G. LeLay, New J. Phys. 16, 095002 (2014).

[9] C.-H. Lin, A. Huang, W. W. Pai, W.-C. Chen, T. Y. Chen, T.-R. Chang, R. Yukawa, C.-M. Cheng, C.-Y. Mou, I. Matsuda, T.-C. Chiang, H.-T. Jeng, and S.-J. Tang, Phys. Rev. Mater. 2, 024003 (2018).

[10] B. Feng, J. Zhang, S. Ito, M. Arita, C. Cheng, L. Chen, K. Wu, F. Komori, O. Sugino, K. Miyamoto, T. Okuda, and S. Meng, Adv. Mater. 30, 1704025 (2018).

[11] B. Feng, O. Sugino, R.-Y. Liu, J. Zhang, R. Yukawa, M. Kawamura, T. Iimori, H. Kim, Y. Hasegawa, H. Li, L. Chen, K. Wu, H. Kumigashira, F. Komori, T.-C. Chiang, S. Meng, and I. Matsuda, Phys. Rev. Lett. 118, 096401 (2017).

[12] B. Feng, J. Zhang, Ro-Ya Liu, T. Iimori, C. Lian, H. Li, L. Chen, K. Wu, S. Meng, F. Komori, and I. Matsuda, Phys. Rev. B 94, 041408(R) (2016).

[13] C. Shekhar, A. N. Nayak, Y. Sun, M. Schmidt, M. Nicklas, I. Leermakers, U. Zeitler, Y. Skourski, J. Wosnitza, Z. Liu, Y. Chen, W. Schnelle, H. Borrman, Y. Grin, C. Felser, and B. Yan, Nat. Phys. 11, 645 (2015).

[14] X. Huang, L. Zhao, Y. Long, P. Wang, D. Chen, Z. Yang, H. Liang, M. Xue, H. Weng, Z. Fang, X. Dai, and G. Chen, Phys. Rev. X 5, 031023 (2015).

[15] Y. Wu, L.-L. Wang, E. Mun, D. D. Johnson, D. Mou, L. Huang, Y. Lee, S. L. Bud'ko, P. C. Canfield, and A. Kaminski, Nat. Phys. 12, 667 (2016).

[16] G. Bian et al., Nat. Commun. 7, 10556 (2016).
[17] L. M. Schoop, M. N. Ali, C. Straßer, A. Topp, A. Varykhalov, D. Marchenko, V. Duppel, S. S. P. Parkin, B. V. Lotsch, and C. R. Ast, Nat. Commun. 7, 11696 (2016).

[18] S. A. Ekahana, S.-C. Wu, J. Jiang, K. Okawa, D. Prabhakaran, C.-C. Hwang, S.-K. Mo, T. Sasagawa, C. Felser, B. Yan, Z. Liu, and Y. Chen, New. J. Phys. 19, 065007 (2017).

[19] B. Feng, B. Fu, S. Kasamatsu, S. Ito, P. Cheng, C.-C. Liu, S. K. Mahatha, P. Sheverdyaeva, P. Moras, M. Arita, O. Sugino, T.-C. Chiang, K. Wu, L. Chen, Y. Yao, and I. Matsuda, Nat. Commun. 8, 1007 (2017).

[20] L. Gao, J.-T. Sun, J.-C. Lu, H. Li, K. Qian, S. Zhang, Y.-Y. Zhang, T. Qian, H. Ding, X. Lin, S. Du, and H.-J. Gao, Adv. Mater. 30, 1707055 (2018).

[21] H. Zhang, Y. Xie, Z. Zhang, C. Zhong, Y. Li, Z. Chen, and Y. Chen, J. Phys. Chem. Lett. 8, 1707 (2017).

[22] S. Gupta, A. Kutana, and B. I. Yakobson, J. Phys. Chem. Lett. 9, 2757 (2018).

[23] T. Mori, H. Borrmann, S. Okada, K. Kudou, A. Leithe-Jasper, U. Burkhardt, and Yu. Grin, Phys. Rev. B 76, 064404 (2007).

[24] S. H. Bauer, J. Am. Chem. Soc. 59, 1096 (1937).

[25] I. Mayer, J. Mol. Struct. 186, 43 (1989).

[26] T. A. Abtew, B. C. Shih, P. Dev, V. H. Crespi, and P. Zhang, Phys. Rev. B 83, 094108 (2011).

[27] Y. Jian, F. Ma, J. Bell, A. Bilic, and A. Du, Angew. Chem. Int. Ed. 55, 10292 (2016).

[28] H. Nishino, T. Fujita, N. T. Cuong, S. Tominaka, M. Miyauchi, S. Iimura, A. Hirata, N. Umezawa, S. Okada, E. Nishibori, A. Fujino, T. Fujimori, S. Ito, J. Nakamura, H. Hosono, and T. Kondo, J. Am. Chem. Soc. 139, 13761 (2017).

[29] I. Tateishi, N. T. Cuong, C. A. S. Moura, M. Cameau, R. Ishibiki, A. Fujino, S. Okada, A. Yamamoto, M. Araki, S. Ito, S. Yamamoto, M. Niibe, T. Tokushima, D. E. Weibel, T. Kondo, M. Ogata, and I. Matsuda, Phys. Rev. Mater. 3, 024004 (2019).

[30] C. Liu, I. Matsuda, R. Hobara, and S. Hasegawa, Phys. Rev. Lett. 96, 036803 (2006).

[31] E. Bianco, S. Butler, S. Jiang, O. D. Restrepo, W. Windl, and J. E. Goldberger, ACS Nano 7, 4414 (2013).

[32] C. J. Bradley and A. P. Cracknell, The Mathematical Theory of Symmetry in Solids: Representation Theory for Point Groups and Space Groups (Oxford University Press, Oxford, 1972).

[33] T. Kariyado and Y. Hatsugai, Phys. Rev. B 88, 245126 (2013)

[34] C. Fang, Y. Chen, H.-Y. Kee, and L. Fu, Phys. Rev. B 92, 081201(R) (2015).

[35] B. J. Wieder and C. L. Kane, Phys. Rev. B 94, 155108 (2016). 
[36] X. Fan, D. Ma, B. Fu, C.-C. Liu, and Y. Yao, Phys. Rev. B 98, 195437 (2018).

[37] P. Hohenberg and W. Kohn, Phys. Rev. 136, B864 (1964).

[38] W. Kohn and L. J. Sham, Phys. Rev. 140, A1133 (1965).

[39] P. Giannozzi et al., J. Phys.: Condens. Matter 21, 395502 (2009).

[40] J. P. Perdew, K. Burke, and M. Ernzerhof, Phys. Rev. Lett. 77, 3865 (1996).

[41] A. D. Corso, Comput. Mater. Sci. 95, 337 (2014).
[42] H. J. Monkhorst and J. D. Pack, Phys. Rev. B 13, 5188 (1976).

[43] A. Togo and I. Tanaka, Scr. Mater. 108, 1 (2015).

[44] International Tables of Crystallography, Space Group Symmetry Vol. A, edited by T. Hahn (Kluwer, Dordrecht, 2002).

[45] S. M. Nie, Z. Song, H. Weng, and Z. Fang, Phys. Rev. B 91, 235434 (2015).

[46] Y. Kim, B. J. Wieder, C. L. Kane, and A. M. Rappe, Phys. Rev. Lett. 115, 036806 (2015). 